\title{
Concave Measures and the Fuzzy Core of Exchange Economies with Heterogeneous Divisible Commodities
}

\author{
Farhad Hüsseinov ${ }^{1}$ Nobusumi Sagara ${ }^{2}$ \\ ${ }^{1}$ Department of Economics, Bilkent University, 06800 Bilkent, Ankara, Turkey \\ ${ }^{2}$ Faculty of Economics, Hosei University, 4342, Aihara, Machida, Tokyo 194-0298, Japan
}

\begin{abstract}
The main purpose of this paper is to prove the existence of the fuzzy core of an exchange economy with a heterogeneous divisible commodity in which preferences of individuals are given by nonadditive utility functions defined on a $\sigma$-algebra of admissible pieces of the total endowment of the commodity. The problem is formulated as the partitioning of a measurable space among finitely many individuals. Applying the Yosida-Hewitt decomposition theorem, we also demonstrate that partitions in the fuzzy core are supportable by prices in $L^{1}$.
\end{abstract}

Keywords: Nonatomic vector measure; Concave measure; Fuzzy coalition; Fuzzy core; Supporting price; Yosida-Hewitt decomposition.

\section{Introduction}

Cooperative fuzzy games proposed by [2, 3] allow for partial participation of individuals in coalitions. In defining the fuzzy core of exchange economies with homogeneous divisible commodities, individuals contribute only some portions of their initial endowments to coalitions they belong to. That is, a fuzzy coalition unlike the classical (crisp) coalitions, does not necessarily require its participants to contribute the whole of their initial endowments. A remarkable result for exchange economies established by [2] states that under the standard assumptions of continuous, convex preferences the fuzzy core and the set of Walrasian allocations coincide (see also $[12,14]$.)

In this paper we study the fuzzy core of an exchange economy with a heterogeneous divisible commodity in which preferences of individuals are given by set functions defined in a $\sigma$-algebra of admissible pieces of the total endowment of the commodity. Following the traditions of fair division literature along the lines of [9], a heterogeneous divisible commodity is modeled as a nonatomic finite measure space. The total endowment of the heterogeneous commodity is metaphorically called a "cake" in this literature and the problem of fair division consists in partitioning the cake among a finite number of individuals according some criteria of fairness and efficiency.
A common assumption in the theory of fair division is that the preferences of each individual are represented by a nonatomic probability measure. Under this additive utility hypothesis, Lyapunov's convexity theorem (see [19]) guarantees the convexity and compactness of the utility possibility set, crucial to establishing the existence and characterization of various solutions. However, here we assume preferences to be represented by nonadditive utility functions; hence the utility possibility set does not necessarily possess these properties. The utility functions assumed here contain concave measures introduced by [20, 21, 22].

$[15,16,23]$ proved the existence of the core with nonadditive evaluations for exchange economies with heterogeneous divisible commodity under diverse assumptions. The corresponding result for the case of the fuzzy core is not straightforward; an adequate notion of fuzzy improvement must reflect awareness of agents of the bounds of the available heterogeneous divisible commodity. We propose here a such notion of the fuzzy improvement and the fuzzy core.

The organization of the paper is as follows: In Section 2 we present a representation result for concave measures, stating that an arbitrary concave measure can be represented as a composition of a concave function and a finite-dimensional nonatomic vector measure. From this characterization we derive the continuity of concave measures at the measurable set whose vector measure lies in the interior of Lyapunov's set. We also provide a core representation theorem for nonatomic vector measure games along the lines of [11].

Section 3 is devoted to the formulation of the fuzzy coalitions and fuzzy core. To this end, we focus our attention on the case where the single heterogeneous divisible commodity possesses a finite number of attributes, which can be evaluated objectively in terms of finite-dimensional nonatomic vector measures. We define the notions of the fuzzy coalitions and fuzzy core in an exchange economy with a heterogeneous divisible commodity, where each individual has a utility function represented by a nonadditive set function.

The main result of this paper, Theorem 4.1 on the existence of the fuzzy core, is stated in Section 
4. To prove this theorem, we extend the commodity space from the set of measurable sets to the set of measurable functions taking values in the unit interval along the lines of $[1,7,10]$. If $f$ in $L^{\infty}$ is a characteristic function of a measurable set $A$, then an individual possessing $f$ is fully entitled to set $A$ and to nothing else. Thus, we can treat allocations in the extended economy with an $L^{\infty}$-commodity space, which can be embedded into the framework of $[5,6]$. We prove the existence of the fuzzy core of this extended economy by constructing a nontransferrable utility (NTU) game and showing that it satisfies the assumptions of Scarf's core existence theorem (see [24]). Exploiting a technique from [18], the existence of the fuzzy core in the original exchange economy follows from the observation that the extreme points in the fuzzy core of the extended economy are indeed measurable partitions.

Section 5 deals with the supportability of efficient partitions by prices. The argument is based on the effective use of the separation theorem under the convexity assumption. We demonstrate that partitions in the fuzzy core are supportable by prices in $L^{1}$, applying the Yosida-Hewitt decomposition theorem (see [25]), which is by now a standard method, having been established by $[5,6]$.

\section{Representation and Continuity of Concave Measures}

\subsection{Representation of Concave Measures}

Let $(\Omega, \mathscr{F})$ be a measurable space with a $\sigma$-algebra $\mathscr{F}$ of subsets of a nonempty set $\Omega$. A measure $\mu$ on $\mathscr{F}$ is nonatomic if, for every $A \in \mathscr{F}$ with $\mu(A)>0$, there exists some $E \in \mathscr{F}$ such that $0<\mu(E)<\mu(A)$. For nonatomic finite measures $\mu_{1}, \ldots, \mu_{m}$, we denote by $\vec{\mu}=\left(\mu_{1}, \ldots, \mu_{m}\right)$ an $\mathbb{R}^{m_{-}}$ valued vector measure. Lyapunov's convexity theorem asserts that the range $\mathscr{R}(\vec{\mu})$ of $\vec{\mu}$ is a compact and convex set in $\mathbb{R}^{m}$ (see [19]).

For an arbitrarily given $A \in \mathscr{F}$ and $t \in[0,1]$, we define the family $\mathscr{K}_{t}^{\vec{\mu}}(A)$ of measurable subsets of $A$ by:

$$
\mathscr{K}_{t}^{\vec{\mu}}(A)=\{E \in \mathscr{F} \mid E \subset A \text { and } \vec{\mu}(E)=t \vec{\mu}(A)\} .
$$

By Lyapunov's convexity theorem, $\mathscr{K}_{t}^{\vec{\mu}}(A)$ is nonempty for every $A \in \mathscr{F}$ and $t \in[0,1]$. Furthermore, for an arbitrarily given $A, B \in \mathscr{F}$ and $t \in[0,1]$, we denote by $\mathscr{K}_{t}^{\vec{\mu}}(A, B)$ the family of sets $C \in \mathscr{F}$, which are written as the union of some disjoint sets $E \in \mathscr{K}_{t}^{\vec{\mu}}(A)$ and $F \in \mathscr{K}_{1-t}^{\vec{\mu}}(B)$. For a nonatomic scalar measure $\mu$, we use $\mathscr{K}_{t}^{\mu}(A, B)$. It is evident that $C \in \mathscr{K}_{t}^{\vec{\mu}}(A, B)$ if and only if $C \in \mathscr{K}_{t}^{\mu_{k}}(A, B)$ for each $k=1, \ldots, m$, and hence, $\mathscr{K}_{t}^{\vec{\mu}}(A, B)=\bigcap_{k=1}^{m} \mathscr{K}_{t}^{\mu_{k}}(A, B)$ for every $A, B \in \mathscr{F}$ and $t \in[0,1]$. It can be shown that $\mathscr{K}_{t}^{\vec{\mu}}(A, B)$ is nonempty for every $A, B \in \mathscr{F}$ and $t \in[0,1]$ (see $[22])$.
The notion of concave measures on $\sigma$-algebras presented in the following definition bears an obvious resemblance to that of concave functions on real vector spaces. We extend here the definition of $[20,21,22]$ to the vector measure case.

Definition 2.1. A set function $\nu: \mathscr{F} \rightarrow \mathbb{R}$ is a concave measure if $\nu(\emptyset)=0$ and there exists a finitedimensional nonatomic vector measure $\vec{\mu}$ such that for every $A, B \in \mathscr{F}$ and $t \in[0,1]$, we have:

$$
t \nu(A)+(1-t) \nu(B) \leqslant \nu(C) \quad \forall C \in \mathscr{K}_{t}^{\vec{\mu}}(A, B) .
$$

When the underlying vector measure is $\vec{\mu}$ for the concave measure $\nu$, we say that $\nu$ is $\vec{\mu}$-concave.

The following result presents a useful representation of concave measures in terms of nonatomic vector measure games along the lines of $[4,11]$.

Theorem 2.1. A set function $\nu: \mathscr{F} \rightarrow \mathbb{R}$ is a concave measure if and only if there exist a finitedimensional nonatomic vector measure $\vec{\mu}$ and a concave function $\varphi: \mathscr{R}(\vec{\mu}) \rightarrow \mathbb{R}$ with $\varphi(0)=0$ such that $\nu=\varphi \circ \vec{\mu}$.

Recall that a set function $\nu: \mathscr{F} \rightarrow \mathbb{R}$ is submodular if $\nu(A \cup B)+\nu(A \cap B) \leqslant \nu(A)+\nu(B)$ for every $A, B \in \mathscr{F}$. The next example is due to [23].

Example 2.1. Let $\mu$ be a nonatomic scalar measure. Define the set function $\nu_{\varphi}: \mathscr{F} \rightarrow \mathbb{R}$ for a continuous function $\varphi: \mathscr{R}(\mu) \rightarrow \mathbb{R}$ with $\varphi(0)=0$ by $\nu_{\varphi}=\varphi \circ \mu$. The following conditions are equivalent:

(i) $\varphi$ is concave;

(ii) $\nu_{\varphi}$ is $\mu$-concave;

(iii) $\nu_{\varphi}$ is submodular.

\subsection{Continuity of Concave Measures}

Sets $A$ and $B$ in $\mathscr{F}$ are $\vec{\mu}$-equivalent if $\vec{\mu}(A \triangle B)=0$, where $A \triangle B=(A \cup B) \backslash(A \cap B)$ is the symmetric difference of $A$ and $B$. The $\vec{\mu}$-equivalence defines an equivalence relation (reflexive, symmetric, transitive binary relation) on $\mathscr{F}$. We denote the $\vec{\mu}$ equivalence class of $A \in \mathscr{F}$ by $[A]$ and denote the set of $\vec{\mu}$-equivalence classes in $\mathscr{F}$ by $\mathscr{F}_{\vec{\mu}}$. Define the metric $d_{\vec{\mu}}$ on $\mathscr{F}_{\vec{\mu}}$ by $d_{\vec{\mu}}([A],[B])=\|\vec{\mu}(A \triangle B)\|$, where $\|\cdot\|$ is the Euclidean norm of $\mathbb{R}^{m}$. If $\mathscr{F}$ is countably generated, then the metric space $\left(\mathscr{F}_{\vec{\mu}}, d_{\vec{\mu}}\right)$ is complete and separable (see [8, Lemma III.7.1]; [13, Theorem 40.B]).

Continuous functions on $\left(\mathscr{F}_{\vec{\mu}}, d_{\vec{\mu}}\right)$ arise in a natural way from the set functions on $\mathscr{F}$. The following definition is a straightforward generalization of $[20,21]$ to the vector measure case.

Definition 2.2. A set function $\nu: \mathscr{F} \rightarrow \mathbb{R}$ is $\vec{\mu}$ continuous at $A \in \mathscr{F}$ if for every $\varepsilon>0$ there exists some $\delta>0$ such that $\|\vec{\mu}(A \triangle B)\|<\delta$ implies $|\nu(A)-\nu(B)|<\varepsilon$. When $\nu$ is $\vec{\mu}$-continuous at every element of $\mathscr{F}$, we say that $\nu$ is $\vec{\mu}$-continuous. 
We denote by $\operatorname{int} \mathscr{R}(\vec{\mu})$ and bd $\mathscr{R}(\vec{\mu})$ the interior of $\mathscr{R}(\vec{\mu})$ and the boundary of $\mathscr{R}(\vec{\mu})$, respectively.

Corollary 2.1. Every concave measure is $\vec{\mu}$-continuous at every $A \in \mathscr{F}$ with $\vec{\mu}(A) \in \operatorname{int} \mathscr{R}(\vec{\mu})$ for some finite-dimensional nonatomic vector measure $\vec{\mu}$.

We denote by $b a(\Omega, \mathscr{F})$ the space of bounded, finitely additive, signed measures on $\mathscr{F}$. A set function $\nu: \mathscr{F} \rightarrow \mathbb{R}$ is a game if $\nu(\emptyset)=0$. A feasible payoff of a game $\nu$ is an element $\mu$ in $b a(\Omega, \mathscr{F})$ satisfying $\mu(\Omega)=\nu(\Omega)$. The core of a game $\nu$ is defined by:

$$
\mathscr{C}(\nu)=\{\mu \in b a(\Omega, \mathscr{F}) \mid \nu \leqslant \mu \text { and } \mu(\Omega)=\nu(\Omega)\},
$$

that is, the core is the set of feasible payoffs upon which no coalition can improve.

Recall that a supergradient of a concave function $\varphi: \mathscr{R}(\vec{\mu}) \rightarrow \mathbb{R}$ at $x \in \mathscr{R}(\vec{\mu})$ is a vector $p \in \mathbb{R}^{m}$ satisfying $\varphi(y)-\varphi(x) \leqslant\langle p, y-x\rangle$ for every $y \in \mathscr{R}(\vec{\mu})$, where $\langle\cdot, \cdot\rangle$ is the inner product in $\mathbb{R}^{m}$. The $s u$ perdifferential $\partial \varphi(x)$ of $\varphi$ at $x$ is the set of supergradients of $\varphi$ at $x$.

Theorem 2.2. If $\nu: \mathscr{F} \rightarrow \mathbb{R}$ is a $\vec{\mu}$-concave measure that is $\vec{\mu}$-continuous at $\Omega$, then there exists a concave function $\varphi: \mathscr{R}(\vec{\mu}) \rightarrow \mathbb{R}$ with $\varphi(0)=0$ such that:

$\mathscr{C}(\nu)=\left\{\begin{array}{l|l}\langle p, \vec{\mu}\rangle \in b a(\Omega, \mathscr{F}) & \begin{array}{l}p \in \partial \varphi(\vec{\mu}(\Omega)) \\ \langle p, \vec{\mu}(\Omega)\rangle=\varphi(\vec{\mu}(\Omega))\end{array}\end{array}\right\}$.

Theorem 2.2 involves a "core representation" result for $\vec{\mu}$-concave measures. Indeed, the core of a $\vec{\mu}$-concave measure $\nu=\varphi \circ \vec{\mu}$ that is $\vec{\mu}$-continuous at $\Omega$ can be characterized by the local behavior of the superdifferential of $\varphi$ at $\vec{\mu}(\Omega)$. A similar characterization of the core of a game with the form $\nu=\varphi \circ \vec{\mu}$ is obtained by [11] under the alternative continuity hypothesis.

\section{Fuzzy Coalitions in Exchange Economies}

\subsection{Partitioning of a Measurable Space}

The problem of dividing of a heterogeneous commodity among a finite number of individuals is formulated as partitioning a measurable space $(\Omega, \mathscr{F})$. Here, set $\Omega$ is a heterogeneous divisible commodity, and $\sigma$-algebra $\mathscr{F}$ of subsets of $\Omega$ describes the collection of possible pieces of $\Omega$. There are $m$ attributes for the heterogeneous divisible commodity $\Omega$, each of which has a cardinal evaluation represented by a nonatomic finite measure $\mu_{k}$ on $(\Omega, \mathscr{F})$ for $k=1, \ldots, m$. Let $\vec{\mu}=\left(\mu_{1}, \ldots, \mu_{m}\right)$ be an $m$ dimensional nonatomic vector measure.

There are $n$ individuals, indexed by $i=1, \ldots, n$, with the set $N=\{1, \ldots, n\}$ of all individuals, whose preferences on $\mathscr{F}$ are given by utility functions $\nu_{i}: \mathscr{F} \rightarrow \mathbb{R}$ for $i \in N$. A partition of $\Omega$ is an ordered $n$-tuple $\left(A_{1}, \ldots, A_{n}\right)$ of mutually disjoint elements
$A_{1}, \ldots, A_{n}$ in $\mathscr{F}$ whose union is $\Omega$, where each $A_{i}$ is a piece of the cake given to individual $i$. Let individual $i$ be initially endowed with $\Omega_{i} \in \mathscr{F}$ for $i \in N$. So $\left(\Omega_{1}, \ldots, \Omega_{n}\right)$ is an initial partition of $\Omega$. An exchange economy $\mathscr{E}=\left\langle(\Omega, \mathscr{F}), \Omega_{i}, \nu_{i}\right\rangle_{i \in N}$ for the partitioning problem under study is the primitive consisting of a common consumption set $(\Omega, \mathscr{F})$ and the individuals' profile of initial endowments $\Omega_{i}$ and utility functions $\nu_{i}$.

We formulate partial participation of individuals to coalitions as proposed by $[2,3]$. A nonzero vector $\alpha=\left(\alpha_{1}, \ldots, \alpha_{n}\right)$ in the unit cube $[0,1]^{n}$ is called a fuzzy coalition, whose component $\alpha_{i} \in[0,1]$ denotes the degree of participation of individual $i$ in this coalition. For each nonempty set $S \subset N$, a fuzzy coalition with support $S$ is a vector $\alpha^{S}=$ $\left(\alpha_{1}^{S}, \ldots, \alpha_{n}^{S}\right) \in[0,1]^{n}$, satisfying $\alpha_{i}^{S}>0$ for each $i \in S$ and $\alpha_{i}^{S}=0$ otherwise; $S$ is the set of 'active individuals' in the fuzzy coalition $\alpha^{S}$. The vector $e^{S}$ in $\{0,1\}^{n}$ defined as $e_{i}^{S}=1$ for each $i \in S$ and $e_{i}^{S}=0$ otherwise is called a crisp coalition, and is identified with an ordinary (nonfuzzy) coalition $S$.

Definition 3.1. A partition $\left(A_{1}, \ldots, A_{n}\right)$ is an $\alpha^{S}$. partition if for each $i \in S$ there exist $E_{i} \in \mathscr{K}_{\alpha_{i}^{S}}^{\vec{\mu}}\left(A_{i}\right)$ and $F_{i} \in \mathscr{K}_{\alpha_{i}^{S}}^{\vec{\mu}}\left(\Omega_{i}\right)$ such that:

$$
\vec{\mu}\left(\bigcup_{i \in S} E_{i} \triangle \bigcup_{i \in S} F_{i}\right)=0 .
$$

An $e^{S}$-partition is simply said to be an $S$-partition.

It follows from the definition that an $S$-partition $\left(A_{1}, \ldots, A_{n}\right)$ satisfies the coalitional feasibility constraint characterwise, that is:

$$
\mu_{k}\left(\bigcup_{i \in S} A_{i} \triangle \bigcup_{i \in S} \Omega_{i}\right)=0
$$

for each $k=1, \ldots, m$.

Definition 3.2. A fuzzy coalition $\alpha^{S}$ improves upon a partition $\left(B_{1}, \ldots, B_{n}\right)$ if there exists an $\alpha^{S_{-}}$ partition $\left(A_{1}, \ldots, A_{n}\right)$ such that $\nu_{i}\left(A_{i}\right)>\nu_{i}\left(B_{i}\right)$ for each $i \in S$. A partition that cannot be improved upon by any fuzzy coalition is a fuzzy core partition.

\subsection{Allocations in $L^{\infty}$-Spaces}

Define $\mu=\sum_{k=1}^{m} \mu_{k}$. Let $L^{\infty}(\Omega, \mathscr{F}, \mu)$ be the space of $\mu$-essentially bounded measurable functions on $\Omega$ with the sup norm. Denote by $\chi_{A} \in L^{\infty}(\Omega, \mathscr{F}, \mu)$ the characteristic function of $A \in \mathscr{F}$.

Let $X=\left\{f \in L^{\infty}(\Omega, \mathscr{F}, \mu) \mid 0 \leqslant f \leqslant 1, \mu\right.$-a.e. $\}$. Then, $X$ is a weakly* compact, convex subset of $L^{\infty}(\Omega, \mathscr{F}, \mu)$. We identify $\mathscr{F}$ with the subset of characteristic functions in $X$. An $n$-tuple $\left(f_{1}, \ldots, f_{n}\right)$ of elements in $L^{\infty}(\Omega, \mathscr{F}, \mu)$ is an allocation of $\Omega$ if $\sum_{i=1}^{n} f_{i}=1$ and $f_{i} \in X$ for each $i \in N$. Note that $\left(A_{1}, \ldots, A_{n}\right)$ is a partition of $\Omega$ if 
and only if $\sum_{i=1}^{n} \chi_{A_{i}}=1$. We denote by $\mathscr{A}$ the set of allocations of $\Omega$.

For $f \in X$, set $\mu_{k}(f)=\int f d \mu_{k}$ for $k=1, \ldots, m$. Given a utility function $\nu_{i}$ of the form $\nu_{i}=\varphi_{i} \circ \vec{\mu}$ with $\varphi_{i}: \mathscr{R}(\vec{\mu}) \rightarrow \mathbb{R}$, we will denote by $\hat{\nu}_{i}$ the extension of $\nu_{i}$ to $X$ defined as $\hat{\nu}_{i}(f)=\varphi_{i}(\vec{\mu}(f))$. This extension is indeed well defined because $\mathscr{R}(\vec{\mu})$ coincides with the set $\{\vec{\mu}(f) \mid f \in X\}$ by Lyapunov's convexity theorem. If $\varphi_{i}$ is continuous and quasiconcave on $\mathscr{R}(\vec{\mu})$, then $\hat{\nu}_{i}$ is weakly* continuous and quasiconcave on $X$.

An exchange economy $\widehat{\mathscr{E}}=\left\langle X, \chi_{\Omega_{i}}, \hat{\nu}_{i}\right\rangle_{i \in N}$ for the allocation problem is the primitive consisting of a common consumption set $X$ and the individuals' profile of initial endowments $\chi_{\Omega_{i}}$ and utility functions $\hat{\nu}_{i}$, which is an extension of the original economy $\mathscr{E}=\left\langle(\Omega, \mathscr{F}), \Omega_{i}, \nu_{i}\right\rangle_{i \in N}$ whenever $\nu_{i}=\varphi_{i} \circ \vec{\mu}$ for each $i \in N$.

Lemma 3.1. $\mathscr{A}$ is a weakly* compact, convex subset of $\left[L^{\infty}(\Omega, \mathscr{F}, \mu)\right]^{n}$.

To explore the notion of fuzzy core allocations for $\widehat{\mathscr{E}}=\left\langle X, \chi_{\Omega_{i}}, \hat{\nu}_{i}\right\rangle_{i \in N}$, we introduce a set-valued mapping $\widehat{\mathscr{K}}_{t}^{\vec{\mu}}: X \rightarrow 2^{X}$, an eligible extension of $\mathscr{K}_{t}^{\vec{\mu}}: \mathscr{F} \rightarrow 2^{\mathscr{F}}$, as follows. For $f \in X$ and $t \in[0,1]$, we define

$$
\widehat{\mathscr{K}}_{t}^{\vec{\mu}}(f)=\{v \in X \mid \vec{\mu}(v)=t \vec{\mu}(f), v \leqslant f\} .
$$

It follows from the definition that $\mathscr{K}_{t}^{\vec{\mu}}(A) \subset$ $\widehat{\mathscr{K}}_{t}^{\vec{\mu}}\left(\chi_{A}\right)$ for every $A \in \mathscr{F}$ and $t \in[0,1]$. Moreover, $\chi_{E} \in \widehat{\mathscr{K}}_{t}^{\vec{\mu}}\left(\chi_{A}\right)$ if and only if $E \in \mathscr{K}_{t}^{\vec{\mu}}(A)$.

Definition 3.3. An allocation $\left(f_{1}, \ldots, f_{n}\right)$ is an $\alpha^{S}$-allocation if for each $i \in S$ there exist $v_{i} \in$ $\widehat{\mathscr{K}}_{\alpha_{i}^{S}}^{\vec{\mu}}\left(f_{i}\right)$ and $w_{i} \in \widehat{\mathscr{K}}_{\alpha_{i}^{S}}^{\vec{\mu}}\left(\chi_{\Omega_{i}}\right)$ such that $\sum_{i \in S} v_{i}=$ $\sum_{i \in S} w_{i}$. An $e^{S}$-allocation is simply said to be an $S$-allocation.

Note that $\left(\chi_{A_{1}}, \ldots, \chi_{A_{n}}\right)$ is an $\alpha^{S}$-allocation with $\left(\chi_{E_{i}}, \chi_{F_{i}}\right) \in \widehat{\mathscr{K}}_{\alpha_{i}^{S}}^{\vec{\mu}}\left(\chi_{A_{i}}\right) \times \widehat{\mathscr{K}}_{\alpha_{i}^{S}}^{\vec{\mu}}\left(\chi_{\Omega_{i}}\right)$ for each $i \in S$ if and only if $\left(A_{1}, \ldots, A_{n}\right)$ is an $\alpha^{S}$-partition with $\left(E_{i}, F_{i}\right) \in \mathscr{K}_{\alpha_{i}^{S}}^{\vec{\mu}}\left(A_{i}\right) \times \mathscr{K}_{\alpha_{i}^{S}}^{\vec{\mu}}\left(\Omega_{i}\right)$ for each $i \in S$. Thus, the notion of $\alpha^{S}$-allocations introduced here is a consistent extension of that of $\alpha^{S}$-partitions to $L^{\infty}$ spaces.

Definition 3.4. A fuzzy coalition $\alpha^{S}$ improves upon an allocation $\left(f_{1}, \ldots, f_{n}\right)$ if there exists an $\alpha^{S_{-}}$ allocation $\left(g_{1}, \ldots, g_{n}\right)$ such that $\hat{\nu}_{i}\left(f_{i}\right)<\hat{\nu}_{i}\left(g_{i}\right)$ for each $i \in S$. An allocation that cannot be improved upon by any fuzzy coalition is a fuzzy core allocation.

When commodities are homogeneous and divisible as in classical exchange economies, the usual definition of an $\alpha^{S}$-allocation is an allocation $\left(f_{1}, \ldots, f_{n}\right)$ such that:

$$
\sum_{i \in S} \alpha_{i}^{S} f_{i}=\sum_{i \in S} \alpha_{i}^{S} \chi_{\Omega_{i}}
$$

(See $[2,3,12,14]$.) Although the definition of $\alpha^{S}$ allocations in the sense of (3.1) seems to make sense in the extended economy $\widehat{\mathscr{E}}$, it is inadequate in that it cannot be reduced to the corresponding definition of $\alpha^{S}$-partitions in the original economy $\mathscr{E}$.

To illustrate how this definition malfunctions, consider for $n=2$ any fuzzy coalition $\alpha=$ $\left(\alpha_{1}, \alpha_{2}\right) \in[0,1]^{2}$ with $\alpha_{1} \neq \alpha_{2}$. Restricting the definition of $\alpha$-allocations in the sense of (3.1) to the characteristic functions $\left(\chi_{A_{1}}, \chi_{A_{2}}\right)$ with $\chi_{A_{1}}+$ $\chi_{A_{2}}=1$ yields $\alpha_{1}\left(\chi_{A_{1}}-\chi_{\Omega_{1}}\right)=\alpha_{2}\left(\chi_{\Omega_{2}}-\chi_{A_{2}}\right)$, which is true if and only if $A_{1}=\Omega_{1}$ and $A_{2}=\Omega_{2}$. So, every $\alpha$-partition with $\alpha_{1} \neq \alpha_{2}$ in the sense of (3.1) consists of only the initial partition $\left(\Omega_{1}, \Omega_{2}\right)$. Fuzziness entirely disappears from the definition.

\section{Existence of Fuzzy Core Allocations}

\subsection{The NTU Game for Exchange Economies}

Let $\mathscr{N}=2^{N} \backslash\{\emptyset\}$. The market game $V: \mathscr{N} \rightarrow$ $2^{\mathbb{R}^{n}}$ with NTU for the exchange economy $\widehat{\mathscr{E}}=$ $\left\langle X, \chi_{\Omega_{i}}, \hat{\nu}_{i}\right\rangle_{i \in N}$ is given by:

$V(S)=\left\{\begin{array}{l|l}x \in \mathbb{R}^{n} & \begin{array}{l}\exists \alpha^{S} \text {-allocation }\left(f_{1}, \ldots, f_{n}\right) \\ \text { such that } x_{i} \leqslant \hat{\nu}_{i}\left(f_{i}\right), \forall i \in S\end{array}\end{array}\right\}$.

By construction, $V(S)$ is the utility possibility set of the players (individuals) in which payoff vectors are attainable via some fuzzy coalition $\alpha^{S}$. The core $C(V)$ of $V$ is given by:

$C(V)=\left\{\begin{array}{l|l}x \in V(N) & \begin{array}{l}\nexists(S, y) \in \mathscr{N} \times V(S) \\ \text { such that } x_{i}<y_{i}, \forall i \in S\end{array}\end{array}\right\}$.

Proposition 4.1. For an exchange economy $\mathscr{E}=$ $\left\langle(\Omega, \mathscr{F}), \Omega_{i}, \nu_{i}\right\rangle_{i \in N}$, if $\nu_{i}$ is of the form $\nu_{i}=\varphi_{i} \circ \vec{\mu}$ such that $\varphi_{i}: \mathscr{R}(\vec{\mu}) \rightarrow \mathbb{R}$ is continuous and quasiconcave for each $i \in N$, then $C(V)$ is nonempty.

Corollary 4.1. For an exchange economy $\mathscr{E}=$ $\left\langle(\Omega, \mathscr{F}), \Omega_{i}, \nu_{i}\right\rangle_{i \in N}$, if $\nu_{i}$ is of the form $\nu_{i}=\varphi_{i} \circ \vec{\mu}$ such that $\varphi_{i}: \mathscr{R}(\vec{\mu}) \rightarrow \mathbb{R}$ is continuous and quasiconcave for each $i \in N$, then there exists a fuzzy core allocation for $\widehat{\mathscr{E}}=\left\langle X, \chi_{\Omega_{i}}, \hat{\nu}_{i}\right\rangle_{i \in N}$.

\subsection{Existence of Fuzzy Core Partitions}

Let $x=\left(x_{1}, \ldots, x_{n}\right)$ be in $C(V)$ and define the set $\mathscr{C}_{x}$ by:

$$
\mathscr{C}_{x}=\left\{\left(f_{1}, \ldots, f_{n}\right) \in \mathscr{A} \mid x_{i} \leqslant \hat{\nu}_{i}\left(f_{i}\right), \forall i \in N\right\} .
$$

It is easy to verify that $\mathscr{C}_{x}$ is a subset of the set of fuzzy core allocations for $\widehat{\mathscr{E}}=\left\langle X, \chi_{\Omega_{i}}, \hat{\nu}_{i}\right\rangle_{i \in N}$.

The proof of the next result is essentially based on the ingenious technique of [18], yielding that an extreme point of $\mathscr{C}_{x}$ is indeed a measurable partition of $\Omega$ (see also [1]).

Proposition 4.2. For an exchange economy $\mathscr{E}=$ $\left\langle(\Omega, \mathscr{F}), \Omega_{i}, \nu_{i}\right\rangle_{i \in N}$, if $\nu_{i}$ is of the form $\nu_{i}=\varphi_{i} \circ \vec{\mu}$ 
such that $\varphi_{i}: \mathscr{R}(\vec{\mu}) \rightarrow \mathbb{R}$ is continuous and quasiconcave for each $i \in N$, then there exists a partition $\left(A_{1}, \ldots, A_{n}\right)$ of $\Omega$ such that $\left(\chi_{A_{1}}, \ldots, \chi_{A_{n}}\right) \in \mathscr{C}_{x}$.

The next theorem is now an immediate consequence of Proposition 4.2 and Corollary 4.1.

Theorem 4.1. For an exchange economy $\mathscr{E}=$ $\left\langle(\Omega, \mathscr{F}), \Omega_{i}, \nu_{i}\right\rangle_{i \in N}$, if $\nu_{i}$ is of the form $\nu_{i}=\varphi_{i} \circ \vec{\mu}$ such that $\varphi_{i}: \mathscr{R}(\vec{\mu}) \rightarrow \mathbb{R}$ is continuous and quasiconcave for each $i \in N$, then there exists a fuzzy core partition.

Corollary 4.2. For an exchange economy $\mathscr{E}=$ $\left\langle(\Omega, \mathscr{F}), \Omega_{i}, \nu_{i}\right\rangle_{i \in N}$, if $\nu_{i}$ is $\vec{\mu}$-concave and $\vec{\mu}$-continuous at every $A \in \mathscr{F}$ with $\vec{\mu}(A) \in \mathrm{bd} \mathscr{R}(\vec{\mu})$ for each $i \in N$, then there exists a fuzzy core partition.

Since a fuzzy core partition is a core partition, Theorem 4.1 is an extension of [15], and [23], the former proved the existence of core partitions for the case where utility functions of the individuals are continuous quasiconcave transformations of a finitedimensional nonatomic vector measure and the latter for the case of concave measures.

\section{Supporting Prices}

\subsection{Continuous, Quasiconcave, Strictly Monotonic Extensions}

For vectors $x, y \in \mathbb{R}^{m}$, denote $x \leqslant y$ to mean that $x_{k} \leqslant y_{k}$ for each $k=1, \ldots, m$ and $x<y$ to mean that $x_{k}<y_{k}$ for each $k=1, \ldots, m$. The positive and strictly positive orthants of $\mathbb{R}^{m}$ are given respectively by $\mathbb{R}_{+}^{m}=\left\{x \in \mathbb{R}^{m} \mid x \geqslant 0\right\}$ and $\mathbb{R}_{++}^{m}=\left\{x \in \mathbb{R}^{m} \mid x>0\right\}$. A function $\varphi: \mathscr{R}(\vec{\mu}) \rightarrow \mathbb{R}$ is monotonic if $x \leqslant y$ and $x, y \in \mathscr{R}(\vec{\mu})$ imply $\varphi(x) \leqslant \varphi(y) ; \varphi$ is strictly monotonic if $x<y$ and $x, y \in \mathscr{R}(\vec{\mu})$ imply $\varphi(x)<\varphi(y)$.

Lemma 5.1. If $\varphi: \mathscr{R}(\vec{\mu}) \rightarrow \mathbb{R}$ is continuous, quasiconcave and strictly monotonic, then $\varphi$ has an extension $\hat{\varphi}: \mathbb{R}_{+}^{m} \rightarrow \mathbb{R}$ preserving its properties.

Since we have assumed that the component measures of $\vec{\mu}=\left(\mu_{1}, \ldots, \mu_{m}\right)$ are mutually absolutely continuous, for every $x \in \mathscr{R}(\vec{\mu})$ with $x \neq \vec{\mu}(\Omega)$, we have $x<\vec{\mu}(\Omega)$. Taking into account this observation, it is helpful to deduce the above lemma from the more general assertion below.

Proposition 5.1. Let $c=\left(c_{1}, \ldots, c_{m}\right) \in \mathbb{R}_{++}^{m}$ and $C$ be a compact convex subset of $\prod_{k=1}^{m}\left[0, c_{k}\right]$ that contains 0 and $c$ satisfying $x<c$ for every $x \in C$ with $x \neq c$. If $\varphi: C \rightarrow \mathbb{R}$ is continuous, quasiconcave and strictly monotonic, then $\varphi$ has an extension $\hat{\varphi}: \mathbb{R}_{+}^{m} \rightarrow \mathbb{R}$ preserving its properties.

If $\varphi: C \rightarrow \mathbb{R}$ is continuous, concave and monotonic, then an extension $\tilde{\varphi}: \mathbb{R}_{+}^{m} \rightarrow \mathbb{R}$ of $\varphi$, preserving its properties, is given by:

$$
\tilde{\varphi}(x)=\max \{\varphi(y) \mid y \in C, y \leqslant x\} .
$$

This extension was suggested by [11]. Unfortunately, when $\varphi$ is continuous, quasiconcave and strictly monotonic, the extension $\tilde{\varphi}$ to $\mathbb{R}_{+}^{m}$ does not necessarily preserve its properties (strict monotonicity might be violated). We have exploited this extension not on the entire domain $\mathbb{R}_{+}^{m}$, but on $D$ in the proof of Proposition 5.1. For a general treatment of extensions of continuous, (strictly) monotonic functions, see [17].

\subsection{Existence of Supporting Prices in $L^{1}$}

Let $b a(\Omega, \mathscr{F}, \mu)$ be the vector subspace of $b a(\Omega, \mathscr{F})$ whose elements vanish at every $A \in \mathscr{F}$ with $\mu(A)=0$. Then, $b a(\Omega, \mathscr{F}, \mu)$ is the dual space of $L^{\infty}(\Omega, \mathscr{F}, \mu)$ (see [8, Theorem IV.8.16]). A nonzero element $\pi \in b a(\Omega, \mathscr{F})$ is positive if $\pi(A) \geqslant 0$ for every $A \in \mathscr{F}$. A positive element $\pi \in b a(\Omega, \mathscr{F})$ is purely finitely additive if every countably additive measure $\lambda$ satisfying $0 \leqslant \lambda \leqslant \pi$ is identically zero.

Definition 5.1. A nonzero element $\pi \in$ $b a(\Omega, \mathscr{F}, \mu)$ is a supporting price for an allocation $\left(f_{1}, \ldots, f_{n}\right)$ for $\widehat{\mathscr{E}}=\left\langle X, \chi_{\Omega_{i}}, \hat{\nu}_{i}\right\rangle_{i \in N}$ if $\hat{\nu}_{i}\left(f_{i}\right) \leqslant \hat{\nu}_{i}(f)$ implies $\pi\left(f_{i}\right) \leqslant \pi(f)$.

As observed by [5, p. 516], "one could call any element of $b a$ a price system, but since those elements of $b a$ not belonging to $L^{1}$ have no economic interpretation, we will be interested only in equilibria with price systems in $L^{1}$." If a supporting price happens to be countably additive, then it has the Radon-Nikodym derivative with respect to $\mu$. In such a case, it is identified with an element in $L^{1}(\Omega, \mathscr{F}, \mu)$.

Definition 5.2. An allocation $\left(f_{1}, \ldots, f_{n}\right)$ in $\widehat{\mathscr{E}}=$ $\left\langle X, \chi_{\Omega_{i}}, \hat{\nu}_{i}\right\rangle_{i \in N}$ is Pareto optimal if there exists no allocation $\left(g_{1}, \ldots, g_{n}\right)$ such that $\hat{\nu}_{i}\left(f_{i}\right) \leqslant \hat{\nu}_{i}\left(g_{i}\right)$ for each $i \in N$ and $\hat{\nu}_{j}\left(f_{j}\right) \leqslant \hat{\nu}_{j}\left(g_{j}\right)$ for some $j \in N$.

Theorem 5.1. For an exchange economy $\mathscr{E}=$ $\left\langle(\Omega, \mathscr{F}), \Omega_{i}, \nu_{i}\right\rangle_{i \in N}$, if $\nu_{i}$ is of the form $\nu_{i}=\varphi_{i} \circ \vec{\mu}$ such that $\varphi_{i}: \mathscr{R}(\vec{\mu}) \rightarrow \mathbb{R}$ is continuous, quasiconcave and strictly monotonic for each $i \in$ $N$, then every Pareto optimal allocation in $\widehat{\mathscr{E}}=$ $\left\langle X, \chi_{\Omega_{i}}, \hat{\nu}_{i}\right\rangle_{i \in N}$ has a positive supporting price.

"[T]heorem [5.1] ... would be of little interest if one could not find interesting conditions under which equilibrium price systems could be chosen from $L^{1} . "$ (See $[5$, p. 523].) To obtain supporting prices in $L^{1}$, we need the following lemma.

Lemma 5.2. For an exchange economy $\mathscr{E}=$ $\left\langle(\Omega, \mathscr{F}), \Omega_{i}, \nu_{i}\right\rangle_{i \in N}$, if $\nu_{i}$ is of the form $\nu_{i}=\varphi_{i} \circ \vec{\mu}$ such that $\varphi_{i}: \mathscr{R}(\vec{\mu}) \rightarrow \mathbb{R}$ is strictly quasiconcave for each $i \in N$, then $\pi \in b a(\Omega, \mathscr{F}, \mu)$ is a supporting price for an allocation $\left(f_{1}, \ldots, f_{n}\right)$ in $\widehat{\mathscr{E}}=\left\langle X, \chi_{\Omega_{i}}, \hat{\nu}_{i}\right\rangle_{i \in N}$ if and only if $\hat{\nu}_{i}\left(f_{i}\right)<\hat{\nu}_{i}(f)$ implies $\pi\left(f_{i}\right) \leqslant \pi(f)$. 
Theorem 5.2. For an exchange economy $\mathscr{E}=$ $\left\langle(\Omega, \mathscr{F}), \Omega_{i}, \nu_{i}\right\rangle_{i \in N}$, if $\nu_{i}$ is of the form $\nu_{i}=\varphi_{i} \circ \vec{\mu}$ such that $\varphi_{i}: \mathscr{R}(\vec{\mu}) \rightarrow \mathbb{R}$ is continuous, strictly quasiconcave and strictly monotonic for each $i \in N$, then every Pareto optimal allocation (in particular, every fuzzy core partition) in $\widehat{\mathscr{E}}=\left\langle X, \chi_{\Omega_{i}}, \hat{\nu}_{i}\right\rangle_{i \in N}$ has a positive supporting price in $L^{1}(\Omega, \mathscr{F}, \mu)$.

\section{References}

[1] E. Akin, Vilfred Pareto cuts the cake, Journal of Mathematical Economics, 24:23-44, 1995.

[2] J.-P. Aubin, Cooperative fuzzy games, Mathematics of Operations Research 6:1-13, 1981.

[3] J.-P. Aubin, Mathematical Methods of Game and Economic Theory, revised edn., NorthHolland, Amsterdam, 1982.

[4] R.J. Aumann and L.S. Shapley, Values of Non-Atomic Games, Princeton University Press, Princeton, 1974.

[5] T.F. Bewley, Existence of equilibria in economies with infinitely many commodities, Journal of Economic Theory, 4:514-540, 1972.

[6] T.F. Bewley, The equality of the core and the set of equilibria in economies with infinitely many commodities and a continuum of agents, International Economic Review, 14:383-394, 1973.

[7] M. Dall'Aglio, The Dubins-Spanier optimization problems in fair division theory, Journal of Computational and Applied Mathematics, 130:17-40, 2001.

[8] N. Dunford and J.T. Schwartz, Linear Operators, Part I: General Theory, John Wiley \& Sons, New York, 1958.

[9] L.E. Dubins and E. H. Spanier, How to cut a cake fairly, American Mathematical Monthly, 68:1-17, 1961.

[10] A. Dvoretsky, A. Wald and J. Wolfowitz, Relations among certain ranges of vector measures, Pacific Journal of Mathematics, 1:59-74, 1951.

[11] E. Einy, D. Moreno and B. Shitovitz, The core of a class of non-atomic games which arise in economic applications, International Journal of Game Theory, 28:1-14, 1999.

[12] M. Florenzano, Edgeworth equilibria, fuzzy core, and equilibria of a production economy without ordered preferences, Journal of Mathematical Analysis and Applications, 153:18-36, 1990.

[13] P. R. Halmos, Measure Theory, Van Nostrand, New York, 1950.

[14] F. Hüsseinov, Interpretation of Aubin's fuzzy coalitions and their extension: Relaxation of finite exchange economics, Journal of Mathematical Economics, 23:499-516, 1994.

[15] F. Hüsseinov, Existence of the core in a heterogeneous divisible commodity exchange economy, International Journal of Game Theory, 37:387-395, 2008.
[16] F. Hüsseinov, A theory of a heterogeneous divisible commodity exchange economy, Journal of Mathematical Economics, 47:54-59, 2011.

[17] F. Hüsseinov, Monotonic extension, Department of Economics, Bilkent University, mimeo., 〈http://www.bilkent.edu.tr/ economics/papers.htm〉, 2010.

[18] J. Lindenstrauss, A short proof of Liapounoff's convexity theorem, Journal of Mathematical Mechanics, 15:971-972, 1966.

[19] A. Lyapunov, A., Sur les fonctions-vecteurs complètement additives, Bull. Acad. Sci. URSS. Sér. Math., 4:465-478, 1940 (in Russian).

[20] N. Sagara and M. Vlach, Representation of preference relations on $\sigma$-algebras of nonatomic measure spaces: Convexity and continuity, Fuzzy Sets and Systems, 160:624-634, 2009.

[21] N. Sagara and M. Vlach, Convex functions on $\sigma$-algebras of nonatomic measure spaces, $\mathrm{Pa}$ cific Journal of Optimization, 6:89-102, 2010.

[22] N. Sagara and M. Vlach, Convexity of the lower partition range of a concave vector measure, Advances in Mathematical Economics, 13:155$160,2010$.

[23] N. Sagara and M. Vlach, A new class of convex games and the optimal partitioning of measurable spaces, forthcoming in International Journal of Game Theory, 〈DOI: 10.1007/s00182010-0258-2), 2010.

[24] H. E. Scarf, The core of an $N$ person game, Econometrica, 35:50-69, 1967.

[25] K. Yosida and E. Hewitt, Finitely additive measures, Transactions American Mathematical Society, 72:46-66, 1952. 